\title{
Recent Results from Reactor Antineutrino Experiments
}

$$
\text { H. Seo }{ }^{1} \text {, }
$$

1 Department of Physics and Astronomy, Seoul National University, Seoul 08826, Korea

* hkseo16@gmail.com

November 26, 2018

Proceedings for the 15th International Workshop on Tau Lepton Physics,
Amsterdam, The Netherlands, 24-28 September 2018
scipost.org/SciPostPhysProc.Tau2018

\begin{abstract}
The smallest neutrino mixing angle $\theta_{13}$ has been successfully measured by the disappearance of reactor antineutrinos at RENO, Daya Bay, and Double Chooz. The oscillation frequency is also measured based on energy and baseline dependent disappearance probability of reactor antineutrinos. Recent results find a variation in the observed reactor antineutrino flux as a function of the reactor fuel evolution. We report more precisely measured values of $\theta_{13}$ and $\Delta m_{e e}^{2}$ and results on the evolution of observed reactor antineutrino yield and spectrum.
\end{abstract}

\section{Contents}

\begin{tabular}{lll}
\hline & Introduction & 1
\end{tabular}

2 Detection Method for Reactor Antineutrinos and Experimental Arrangements 2

3 Precise Measurements of $\theta_{13}$ and $\Delta m_{e e}^{2} \quad 2$

4 Fuel-composition Dependent Reactor Antineutrino Yield and Spectrum 3

5 Conclusion 5

\begin{tabular}{ll}
\hline References & 6 \\
\hline
\end{tabular}

\section{Introduction}

The reactor $\bar{\nu}_{e}$ disappearance has been firmly observed to determine the smallest neutrino mixing angle $\theta_{13}[1][3]$. All of the three mixing angles in the Pontecorvo-Maki-NakagawaSakata matrix [4,5] have been measured to provide a comprehensive picture of neutrino transformation. The successful measurement of a rather large $\theta_{13}$ value opens the possibility of searching for $C P$ violation in the leptonic sector and determining the neutrino 
mass ordering. The appearance of $\nu_{e}$ from an accelerator $\nu_{\mu}$ beam is also observed by the $\mathrm{T} 2 \mathrm{~K}[6]$ and $\mathrm{NO} \nu \mathrm{A}[7]$ experiments. Using the $\bar{\nu}_{e}$ survival probability $P[8$, reactor experiments with a baseline distance of $\sim 1 \mathrm{~km}$ has determined the mixing angle $\theta_{13}$ and an effective squared mass difference $\Delta m_{e e}^{2} \equiv \cos ^{2} \theta_{12} \Delta m_{31}^{2}+\sin ^{2} \theta_{12} \Delta m_{32}^{2}$ [9], based on the rate, spectral and baseline information.

In reactor experiment at distances $<100 \mathrm{~m}$ from the reactor core, the measured rate of $\bar{\nu}_{e}$ was found to be in good agreement with that predicted from the reactor antineutrino spectra derived from $\beta$ spectra at ILL and Vogel's theoretical calculation. However, 2011 reevaluation of the reactor antineutrino flux and spectrum with improved theoretical treatments [10,11] results in small increase in the flux to be higher than the experimental data. The $\sim 6 \%$ deficit of data is called as Reactor Antineutrino Anomaly (RAA) [12]. There have been attempts to explain the anomaly by the existence of sterile neutrinos or incorrect calculation of the expected antineutrino flux. Daya Bay collaboration reported an observation of reactor fuel dependent variation of the reactor $\bar{\nu}_{e}$ flux and spectrum and concluded that the ${ }^{235} \mathrm{U}$ fuel isotope may be the primary contributor to the RAA [13. Recently, RENO collaboration also reported the analysis result on the fuel dependent variation of the reactor $\bar{\nu}_{e}$ flux and spectrum [14]. RENO collaboration showed a hint of correlation between the $5 \mathrm{MeV}$ excess and the ${ }^{235} \mathrm{U}$ fuel isotope fraction.

\section{Detection Method for Reactor Antineutrinos and Exper- imental Arrangements}

Reactor antineutrinos mainly come from the beta decays of fission products of ${ }^{235} \mathrm{U},{ }^{238} \mathrm{U}$, ${ }^{239} \mathrm{Pu},{ }^{241} \mathrm{Pu}$. The reactor $\bar{\nu}_{e}$ is detected through the inverse beta decay (IBD) interaction, $\bar{\nu}_{e}+p \rightarrow e^{+}+n$, with free protons in hydrocarbon liquid scintillator (LS) with $0.1 \%$ gadolinium $(\mathrm{Gd})$ as a target. The coincidence of a prompt positron signal and a mean time of $\sim 28 \mu$ s delayed signal from neutron capture by Gd $(n-\mathrm{Gd})$ provides the distinctive IBD signature against backgrounds. The prompt signal releases energy of $1.02 \mathrm{MeV}$ as two $\gamma$-rays from the positron annihilation in addition to the positron kinetic energy. The delayed signal produces several $\gamma$-rays with the total energy of $\sim 8 \mathrm{MeV}$.

The RENO uses identical near and far $\bar{\nu}_{e}$ detectors located 294 and $1383 \mathrm{~m}$, respectively, from the center of six reactor cores of the Hanbit (known as Yonggwang) Nuclear Power Plant in South Korea. The Daya Bay experiment has two near experimental halls (EH1 and EH2 each housing two detectors) and one far experimental hall (EH3 housing four detectors) at the Daya Bay nuclear power plant consisting of six reactors, in the southern part of China. The Double Chooz experiment has two detectors located at distance of 400 and $1050 \mathrm{~m}$ from the two reactor cores of the Chooz nuclear power plant in France. The three reactor experiments have similar experimental arrangements, but slightly different features in reactor thermal output, detector target mass and overburden, and baselines of near and far detectors.

\section{Precise Measurements of $\theta_{13}$ and $\Delta m_{e e}^{2}$}

Oscillation amplitude and frequency of neutrino survival probability are measured based on the information of the observed reactor neutrino rate and spectra. $\sin ^{2} 2 \theta_{13}$ and $\left|\Delta m_{e e}^{2}\right|$ are determined by comparing measured far-to-near ratio of IBD prompt spectra to that of prediction. RENO was the first reactor experiment to take data with both near and far detectors in operation from August 2011. With the increased statistics of the 2200 day 
data sample and the reduced background rate, the RENO collaboration has observed clear energy dependent disappearance of reactor $\bar{\nu}_{e}$ using two identical detectors, and obtained $\sin ^{2} 2 \theta_{13}=0.0896 \pm 0.0068(7.6 \%)$ and $\left|\Delta m_{e e}^{2}\right|=2.68 \pm 0.14\left(\times 10^{-3} \mathrm{eV}^{2}\right)(5.2 \%)$. The experiment has observed an excess of IBD prompt spectra near $5 \mathrm{MeV}$ with respect to the Huber-Mueller model 10,11 as shown in Figure 1. It is found that the excess come from reactors since it is strongly correlated to the reactor thermal powers. Daya Bay and Double Chooz have also reported the observation of $5 \mathrm{MeV}$ excess in the IBD prompt spectrum and its correlation with reactor thermal powers.
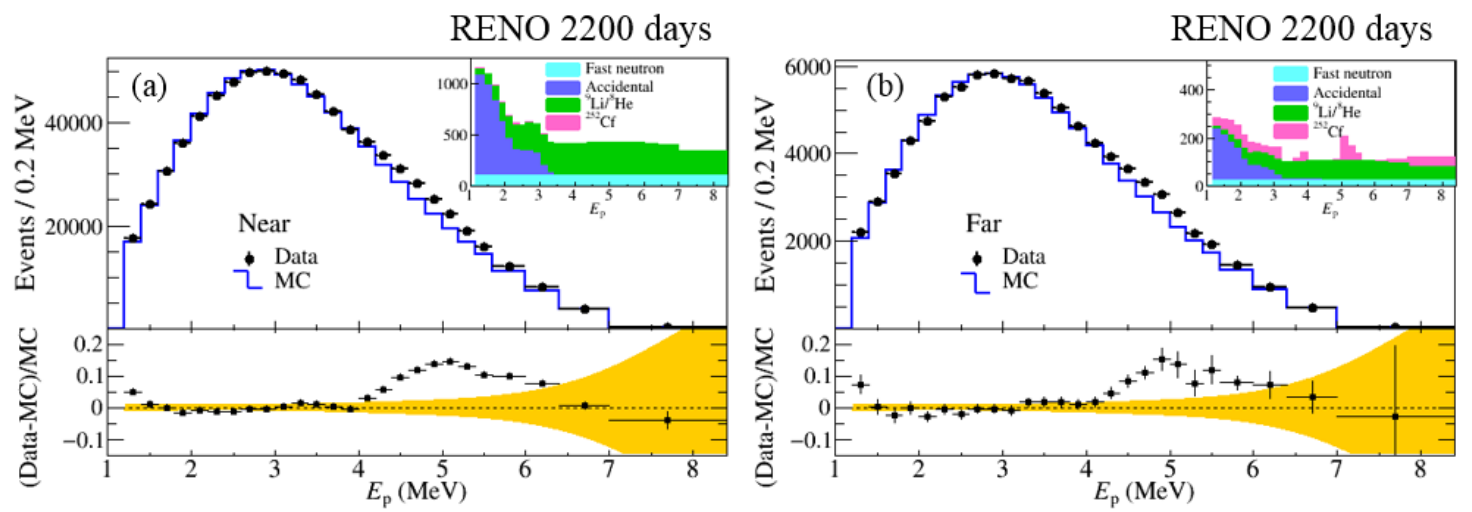

Figure 1: Comparison of observed and expected IBD prompt energy spectrum in the RENO near (a) and far (b) detectors. The expected distributions are obtained from the best-fit oscillation results. The excess at around $5 \mathrm{MeV}$ is clearly seen. A spectral-only comparison is made by normalizing the MC predicted energy spectra to the observed events out of the $5 \mathrm{MeV}$ excess range.

The Daya Bay collaboration has obtained new results on $\theta_{13}$ and $\Delta m_{e e}^{2} \cdot \sin ^{2} 2 \theta_{13}=$ $0.0856 \pm 0.0029(3.4 \%)$ and $\left|\Delta m_{e e}^{2}\right|=2.52 \pm 0.07\left(\times 10^{-3} e^{2}\right)(2.8 \%)$ with 1958 days of data and reduced systematic uncertainties. This is the most precise measurement of $\sin ^{2} 2 \theta_{13}$.

Double Chooz has been taking data with a far detector since April 2011 while the near detector started data taking since January 2015. The result with 818 days of far data and 258 days of near data is $\sin ^{2} 2 \theta_{13}=0.105 \pm 0.014(13 \%)$.

Measured values of $\sin ^{2} 2 \theta_{13}$ and $\Delta m_{32}^{2}$ from various experiments are compared in Figure 2.

\section{Fuel-composition Dependent Reactor Antineutrino Yield and Spectrum}

A study has been on progress to find changes in the observed reactor antineutrino flux and spectrum with respect to the reactor fuel evolution. Through this study, we test reactor antineutrino model and find possible source of RAA. Left hand side plot of Figure 3 shows a measured IBD yield per fission $\bar{y}_{f}$ as a function of the effective fission fraction using RENO near data. RENO observe a clear dependence of the IBD yield per fission on the effective fission fraction. This result rules out no fuel-dependent variation of IBD yield per fission at $6.6 \sigma$. The observed slope of the IBD yield variation over fission fraction deviates from that of Huber-Mueller (HM) model prediction [10,11] by only $1.3 \sigma$, indicating good consistency within experimental uncertainties. $y_{235}$ and $y_{239}$ denoting IBD yield per fission for individual isotope ${ }^{235} \mathrm{U}$ and ${ }^{239} \mathrm{Pu}$, respectively, are determined using the eight data 

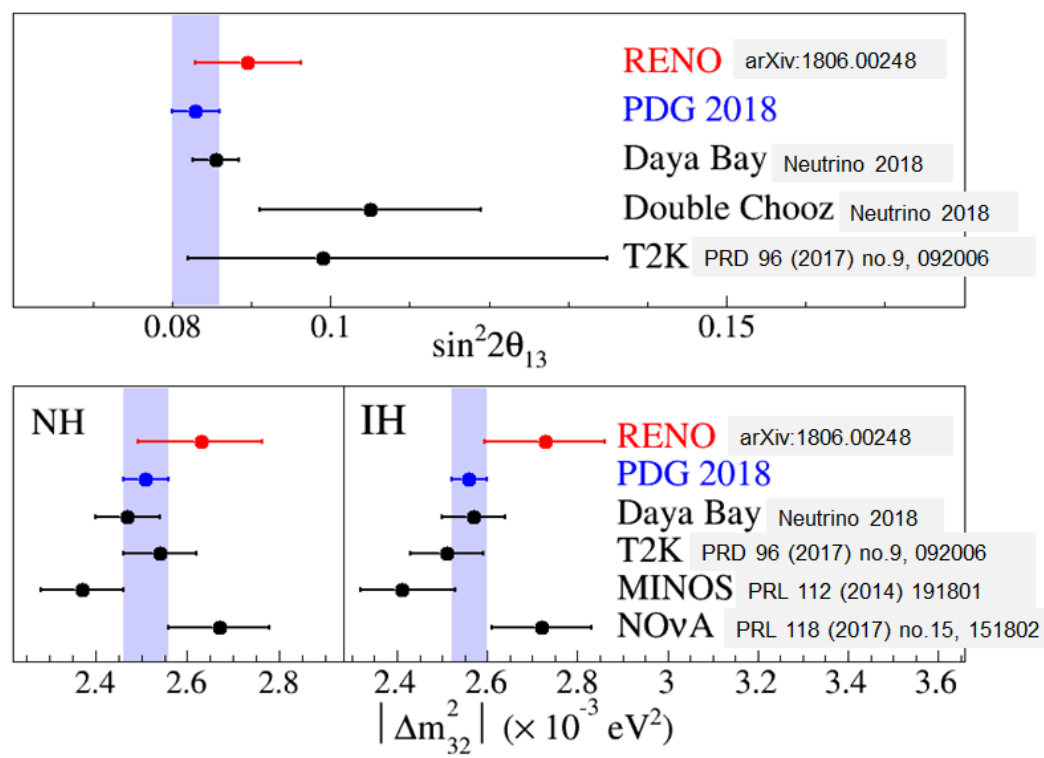

Figure 2: Comparison of experimental results on $\sin ^{2} 2 \theta_{13}$ and $\Delta m_{32}^{2}$.

points of the left hand side plot of Figure 3 by a $\chi^{2}$ fit with pull parameters of systematic uncertainties. Right hand side plot of Figure 3 shows the result of the measurements of $y_{235}$ and $y_{239}$. The contours are allowed regions, the dot is the best fit and the cross is the HM prediction. The measured $y_{235}$ is smaller than the HM prediction at $2.8 \sigma$ while measured $y_{239}$ is smaller than the HM prediction at only $0.8 \sigma$. This suggests the RAA can be largely understood by incorrect prediction of $y_{235}$. However, it is difficult to determine whether ${ }^{235} \mathrm{U}$ alone is responsible for the RAA because the uncertainty for ${ }^{239} \mathrm{Pu}$ is too large.

Similar analysis on the fuel-composition dependent reactor antineutrino yield has also been reported by Daya Bay in 2017 [13]. Daya Bay rules out no fuel-dependent variation of IBD yield per fission at $10 \sigma(6.6 \sigma$ for RENO) and observes the difference between measured IBD yield variation over ${ }^{239} \mathrm{Pu}$ fraction and $\mathrm{HM}$ prediction at $2.6 \sigma(1.3 \sigma$ for RENO). Based on a comparison of the measured and predicted IBD yields per fission for the dominant fission isotopes ${ }^{235} \mathrm{U}$ and ${ }^{239} \mathrm{Pu}$, Daya Bay concluded that the ${ }^{235} \mathrm{U}$ fuel isotope may be the primary contributor to the RAA. To examine a possible difference of fuel dependence between measured IBD yield and $\mathrm{HM}$ prediction at the $5 \mathrm{MeV}$ region, the IBD yield variations over effective fission fraction are obtained for different prompt energy ranges. No significant difference between the measured IBD yield variation over effective fission fraction and HM prediction has been observed within experimental uncertainties for all the prompt energy ranges in both RENO and Daya Bay.

For more sensitive examination of fuel dependence of the $5 \mathrm{MeV}$ excess, RENO obtains an event rate of $5 \mathrm{MeV}$ excess only by subtracting $\mathrm{HM}$ expected energy spectrum in the 5 $\mathrm{MeV}$ region of $3.8<E_{p}<7 \mathrm{MeV}$. The HM prediction in the $5 \mathrm{MeV}$ region is determined by a fit to the data in the region excluding $3.8<E_{p}<7 \mathrm{MeV}$. A fraction of the $5 \mathrm{MeV}$ excess is calculated as a ratio of the $5 \mathrm{MeV}$ excess rate to the total IBD rate. Figure 4 shows the fraction of $5 \mathrm{MeV}$ excess as a function of ${ }^{235} \mathrm{U}$ effective fraction. The best fit for the data shows correlation between the $5 \mathrm{MeV}$ excess fraction and ${ }^{235} \mathrm{U}$ fission fraction. The hypothesis of no correlation between the fraction of $5 \mathrm{MeV}$ excess and ${ }^{235} \mathrm{U}$ fission fraction is disfavored at $2.7 \sigma$. 

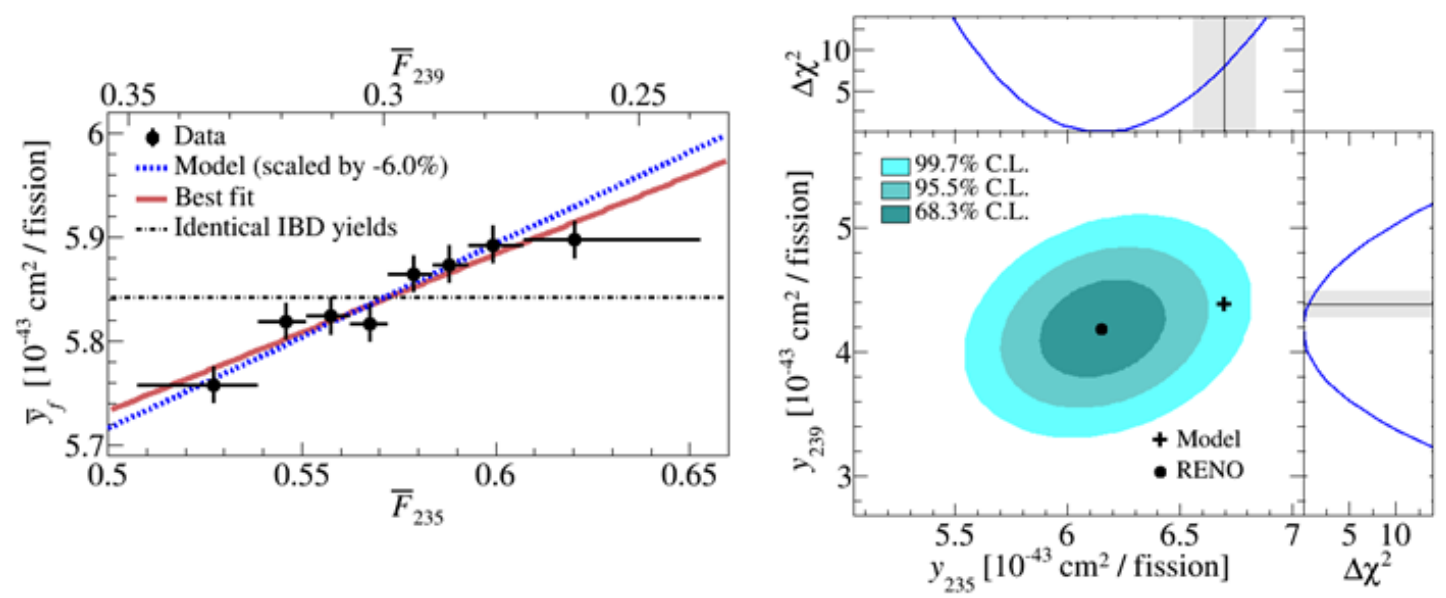

Figure 3: Left: IBD yield per fission $\bar{y}_{f}$ as a function of the effective fission fraction at RENO near detector. The black dots are measured values, blue dotted line is the $-6.0 \%$ scaled Huber-Mueller model prediction and red solid line is the best fit of the data. The horizontal errors of the data indicate the range of effective fission fraction. Errors of $\bar{y}_{f}$ are statistical uncertainties only. Right: measurement of IBD yield per fission for individual isotope ${ }^{235} \mathrm{U}$ and ${ }^{239} \mathrm{Pu}$. The contours are allowed regions, the dot is the best fit and the cross is the Huber-Mueller model prediction.

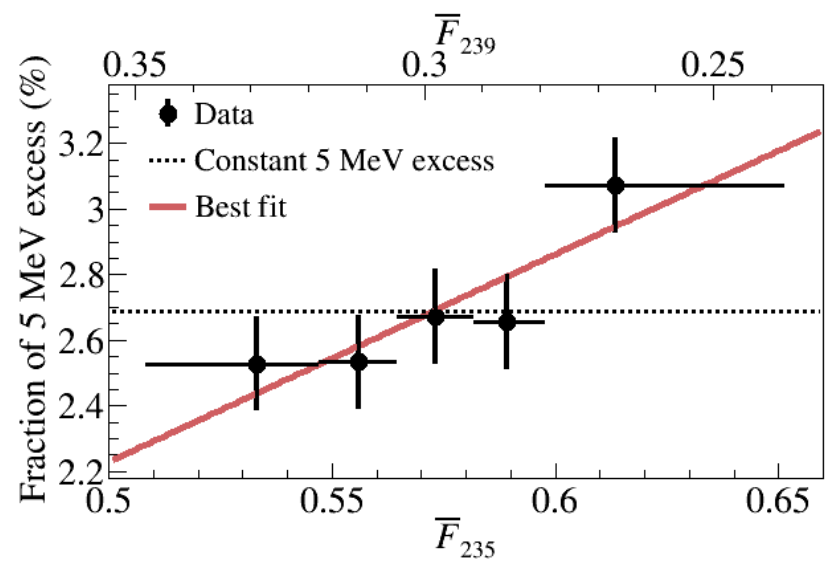

\section{Conclusion}

Reactor antineutrino experiments have observed a clear energy dependent disappearance of reactor $\bar{\nu}_{e}$ at far detectors and updated the results of $\sin ^{2} 2 \theta_{13}$ and $\left|\Delta m_{e e}^{2}\right|$ with increased statistics and reduced systematic uncertainties. The precision measurements provides a comprehensive picture of neutrino transformation among three kinds of neutrinos and open the possibility of search for $\mathrm{CP}$ violation in the leptonic sector. RENO and Daya Bay report a fuel-dependent IBD yield and spectrum. Hypothesis of no fuel-dependent IBD yield is ruled out at $6.6 \sigma(10 \sigma)$ in RENO (Daya Bay). The smaller measured IBD yield per ${ }^{235} \mathrm{U}$ fission than the Huber-Mueller model suggests that the reactor antineutrino anomaly can be largely understood by reevaluation of the ${ }^{235} \mathrm{U}$ IBD yield prediction. RENO obtains the first hint of correlation between the fraction of $5 \mathrm{MeV}$ excess and ${ }^{235} \mathrm{U}$ fission fraction. 


\section{Acknowledgements}

This work is supported by the National Research Foundation of Korea (NRF) Grant No. 2009-0083526 funded by the Korea Ministry of Science, ICT, and Future Planning.

\section{References}

[1] J. K. Ahn et al. (RENO Collaboration), Observation of Reactor Electron Antineutrino Disappearance in the RENO Experiment, Phys. Rev. Lett. 108, 191802 (2012), doi:10.1103/PhysRevLett.108.191802.

[2] F. P. An et al. (Daya Bay Collaboration), Observation of electron-antineutrino disappearance at Daya Bay , Phys. Rev. Lett. 108, 171803 (2012), doi:10.1103/PhysRevLett.108.171803.

[3] Y. Abe et al. (Double Chooz Collaboration), Indication of Reactor $\bar{\nu}_{e}$ Disappearance in the Double Chooz Experiment, Phys. Rev. Lett. 108, 131801 (2012), doi:10.1103/PhysRevLett.108.131801.

[4] B. Pontecorvo, Inverse beta processes and nonconservation of lepton charge, Zh. Eksp. Teor. Fiz. 34, 247 (1957) [Sov. Phys. JETP 7, 172 (1958)].

[5] Z. Maki, M. Nakagawa, and S. Sakata, Remarks on the unified model of elementary particles, Prog. Theor. Phys. 28, 870 (1962), doi:10.1143/PTP.28.870.

[6] K. Abe et al. (T2K Collaboration), Evidence of Electron Neutrino Appearance in a Muon Neutrino Beam, Phys. Rev. D 88, 032002 (2013), doi:10.1103/PhysRevD.88.032002; K. Abe et al. (T2K Collaboration), Observation of Electron Neutrino Appearance in a Muon Neutrino Beam, Phys. Rev. Lett. 112, 061802 (2014), doi:10.1103/PhysRevLett.112.061802.

[7] P. Adamson et al. ( $\mathrm{NO} \nu \mathrm{A}$ Collaboration), First measurement of electron neutrino appearance in NOvA, Phys. Rev. Lett. 116, 151806 (2016), doi:103/PhysRevLett.116.151806.

[8] S. T. Petcov and M. Piai, The LMA MSW solution of the solar neutrino problem, inverted neutrino mass hierarchy and reactor neutrino experiments, Phys. Lett. B 533, 94 (2002), doi:10.1016/S0370-2693(02)01591-5.

[9] H. Nunokawa, S. Parke, and R. Zukanovich Funchal, Another possible way to determine the neutrino mass hierarchy, Phys. Rev. D 72, 013009 (2005), doi:10.1103/PhysRevD.72.013009.

[10] T.A.Mueller et al., Improved Predictions of Reactor Antineutrino Spectra, Phys. Rev. C 83, 054615 (2011), doi:10.1103/PhysRevC.83.054615.

[11] P. Huber, On the determination of anti-neutrino spectra from nuclear reactors, Phys. Rev. C 84, 024617 (2011), doi:10.1103/PhysRevC.84.024617.

[12] G. Mention, M. Fechner, Th. Lasserre, Th. A. Mueller, D. Lhuillier, M. Cribier, and A. Letourneau, The Reactor Antineutrino Anomaly, Phys. Rev. D 83, 073006 (2011), doi:10.1103/PhysRevD.83.073006. 
[13] F. P. An et al. (Daya Bay Collaboration), Evolution of the Reactor Antineutrino Flux and Spectrum at Daya Bay , Phys. Rev. Lett. 118, 251801 (2017), doi:10.1103/PhysRevLett.118.251801.

[14] J. Yoo et al. (RENO Collaboration), Fuel-composition dependent reactor antineutrino yield and spectrum at RENO, https://arxiv.org/abs/1806.00574. 